\title{
Peierls distortion, magnetism, and high hardness of manganese tetraboride
}

\author{
Huiyang Gou, ${ }^{1,2}$ Alexander A. Tsirlin,,${ }^{3,}$ Elena Bykova, ${ }^{1,2}$ Artem M. Abakumov, ${ }^{4}$ Gustaaf Van Tendeloo, ${ }^{4}$ Asta Richter, ${ }^{5}$ \\ Sergey V. Ovsyannikov, ${ }^{1}$ Alexander V. Kurnosov, ${ }^{1}$ Dmytro M. Trots, ${ }^{1}$ Zuzana Konôpková, ${ }^{6}$ Hans-Peter Liermann, ${ }^{6}$ \\ Leonid Dubrovinsky, ${ }^{1}$ and Natalia Dubrovinskaia ${ }^{2, *}$ \\ ${ }^{1}$ Bayerisches Geoinstitut, Universität Bayreuth, D-95440 Bayreuth, Germany \\ ${ }^{2}$ Material Physics and Technology at Extreme Conditions, Laboratory of Crystallography, University of Bayreuth, \\ D-95440 Bayreuth, Germany \\ ${ }^{3}$ National Institute of Chemical Physics and Biophysics, Akadeemia tee 23, E-12618 Tallinn, Estonia \\ ${ }^{4}$ Electron Microscopy for Materials Research (EMAT), University of Antwerp, Groenenborgerlaan 171, B-2020 Antwerp, Belgium \\ ${ }^{5}$ Technische Hochschule Wildau, Bahnhofstrasse 1, D-15745 Wildau, Germany \\ ${ }^{6}$ DESY Photon Science, Deutsches Electronen Synchrtron, Notkestrasse 85, D-22607 Hamburg, Germany
}

(Received 25 December 2013; published 24 February 2014)

\begin{abstract}
We report crystal structure, electronic structure, and magnetism of manganese tetraboride, $\mathrm{MnB}_{4}$, synthesized under high-pressure, high-temperature conditions. In contrast to superconducting $\mathrm{FeB}_{4}$ and metallic $\mathrm{CrB}_{4}$, which are both orthorhombic, $\mathrm{MnB}_{4}$ features a monoclinic crystal structure. Its lower symmetry originates from a Peierls distortion of the Mn chains. This distortion nearly opens the gap at the Fermi level, but despite the strong dimerization and the proximity of $\mathrm{MnB}_{4}$ to the insulating state, we find indications for a sizable paramagnetic effective moment of about $1.7 \mu_{B}$ /f.u., ferromagnetic spin correlations, and, even more surprisingly, a prominent electronic contribution to the specific heat. However, no magnetic order has been observed in standard thermodynamic measurements down to $2 \mathrm{~K}$. Altogether, this renders $\mathrm{MnB}_{4}$ a structurally simple but microscopically enigmatic material; we argue that its properties may be influenced by electronic correlations.
\end{abstract}

DOI: 10.1103/PhysRevB.89.064108

PACS number(s): 61.50.-f, 62.20.-x, 75.20.En

\section{INTRODUCTION}

The "electron-deficient" character of boron and its chemical activity lead to the formation of numerous boron-rich compounds of various structural complexity [1-3]. A plethora of interesting physical and chemical properties of boronbased solids, such as mechanical strength, high hardness, superconductivity, catalytic activity, and thermoelectricity, keeps them in the focus of modern experimental and theoretical research [4-7].

The synthesis of diborides of $5 d$ noble metals, $\mathrm{OsB}_{2}$ and $\mathrm{ReB}_{2}$, was driven by expectations to obtain a new type of superhard materials [7-10] at ambient pressure. However, a careful analysis of the available data [11] and following investigations $[12,13]$ did not confirm the proclaimed superhardness. Unexpected superhardness was found for iron tetraboride $\left(\mathrm{FeB}_{4}\right)$ synthesized at high pressures and temperatures [6], while other transition-metal tetraborides (e.g., $\mathrm{CrB}_{4}$ and $\mathrm{WB}_{4}$ ) $[14,15]$ are hard, but not superhard materials - their hardness is below $30 \mathrm{GPa}$ in the asymptotic hardness region. Moreover, iron tetraboride was found to be superconducting [6], thus possessing a combination of useful properties, which are desirable for a variety of engineering applications. This particular example motivated us for a further exploration of transition-metal tetraborides, in particular, $\mathrm{MnB}_{4}$. Its detailed structure investigation is still missing - the Inorganic Crystal Structure Database (ICSD) provides information about the monoclinic crystal structure of $\mathrm{MnB}_{4}$ (space group $C 2 / \mathrm{m}$ ), ICSD \#15079, based on powder x-ray diffraction data of

\footnotetext{
*Corresponding authors: altsirlin@gmail.com, natalia. dubrovinskaia@uni-bayreuth.de
}

Andersson [16] and Andersson and Carlsson [17] obtained in the late 1960s.

Here, we report the successful synthesis of single crystals of $\mathrm{MnB}_{4}$ at high pressures and temperatures, solution, and refinement of its crystal structure based on single-crystal $\mathrm{x}$ ray diffraction, and results of investigations of the material's compressibility, hardness, magnetic properties, and electronic structure.

\section{MATERIALS AND METHODS}

\section{A. Starting materials and synthesis}

Polycrystalline $\mathrm{MnB}_{4}$ samples were synthesized at highpressure and high-temperature conditions in a piston-cylinder apparatus. Boron (Chempur Inc., 99.99\% purity) and manganese (Alfa Aesar, 99.9\% purity) powders were mixed in a stoichiometric (4:1) ratio. The mixture was loaded into a double capsule consisting of $h$-BN (inner) and Pt (outer) parts and then compressed to $3 \mathrm{GPa}$ and heat treated at either $1080{ }^{\circ} \mathrm{C}, 1350{ }^{\circ} \mathrm{C}$, or $1500^{\circ} \mathrm{C}$. The duration of heating varied from 4 to $240 \mathrm{~h}$. The samples were abruptly quenched by switching off the furnace power.

Pressure calibration was performed prior to the synthesis. It is based on the quartz-coesite and kyanite-sillimanite transitions, as well as on the melting point of diopside. The measured pressure is considered to be accurate within less than $\pm 5 \%$. The temperature was measured with a Pt-Pt $10 \%$ $\mathrm{Rh}$ thermocouple. Temperature gradients are estimated to be less than $25^{\circ} \mathrm{C}$ for the described experimental conditions.

Single crystals of $\mathrm{MnB}_{4}$ were synthesized at pressures of 10 and $12 \mathrm{GPa}$ and a temperature of $1600{ }^{\circ} \mathrm{C}$ (heating duration was $1 \mathrm{~h}$ ) in the Kawai-type multianvil apparatus [18] using 1000-ton (Hymag) and 1200-ton (Sumitomo) hydraulic presses and 14/8 (octahedron edge length/cube truncation 
length) high-pressure assemblies. As starting materials we used a manganese rod (Goodfellow, 99.5\% purity) and a boron powder (Chempur Inc., $99.99 \%$ purity) which were enclosed into a $h$-BN capsule. The pressure was calibrated based on the phase transitions of standard materials and the temperature was determined using a W3Re/W25Re thermocouple.

\section{B. Analytical techniques}

The morphology and chemical composition of the synthesized single crystals were studied by means of scanning electron microscopy (SEM) (LEO-1530). Chemical purity of the samples was confirmed using wavelength dispersive $\mathrm{X}$-ray (WDX) microprobe analysis (JEOL JXA-8200; focused beam; $12 \mathrm{keV}$ and $15 \mathrm{nA}$ or $15 \mathrm{keV}$ and $12 \mathrm{nA}$ ). The LIFH and LDEB crystals were used to analyze $\mathrm{Mn}$ and $\mathrm{B}$, respectively. Pure Mn and $\alpha$-B or FeB were used as internal standards with atomic number effects, absorption, and fluorescence (ZAF) correction.

\section{Single-crystal x-ray diffraction}

A black lustrous thin plate of $\mathrm{MnB}_{4}$ with a size of $0.05 \times 0.04 \times 0.01 \mathrm{~mm}^{3}$ was used for the crystal structure investigation by means of single-crystal $x$-ray diffraction. $\mathrm{X}$-ray diffraction data were collected at ambient temperature using a four-circle Oxford Diffraction Xcalibur diffractometer $(\lambda=0.7107 \AA)$ equipped with an Xcalibur Sapphire 2 chargecoupled device (CCD) detector. The intensities of the reflections were measured by step scans in omega scanning with a narrow step width of $0.5^{\circ}$. The data collection and their further integration were performed with the CRYSALISPRO software [19]. Absorption corrections were applied empirically by the SCALE3 ABSPACK program implemented in CRYSALISPRO. The scaling and absorption corrections were used due to the small size of the inspected crystal that makes precise face indexing difficult. The structure was solved by the direct method and refined by the full matrix least squares in the anisotropic approximation for all atoms using SHELXTL software [20]. The $\mathrm{X}$-ray experimental details and crystallographic characteristics of $\mathrm{MnB}_{4}$ are presented in Table I. The DIAMOND software [21] was used to create molecular graphics.

The crystallographic data of $\mathrm{MnB}_{4}$ and further details of the crystal structure investigation have been deposited in the Inorganic Crystal Structure Database [22] and may be obtained free of charge from Fachinformationszentrum Karlsruhe [23] on quoting the deposition number CSD-426691.

\section{High-pressure powder x-ray diffraction}

For in situ high-pressure x-ray diffraction (XRD) studies we employed a piston-cylinder-type diamond anvil cell with a culet size of $350 \mu \mathrm{m}$ and a rhenium gasket. A small sample $\left(\sim 20 \mu \mathrm{m}\right.$ in size) of $\mathrm{a} \mathrm{MnB}_{4}$ powder was loaded into a hole of $\sim 150 \mu \mathrm{m}$ in diameter drilled in the gasket preindented to $\sim 50 \mu \mathrm{m}$. Using a gas-loading apparatus at Bayerisches Geoinstitut (BGI) [24], we loaded the pressure chamber with the sample along with neon as pressure-transmitting medium. The XRD experiments were carried out at the Extreme Conditions Beamline (ECB) P02.2 at PETRA III, DESY (Hamburg) [25]. The x-ray wavelength was $\lambda=0.29135 \AA$. The pressure
TABLE I. Details on the x-ray diffraction data collection and structure refinement of $\mathrm{MnB}_{4}$.

\begin{tabular}{|c|c|}
\hline Empirical formula & $\mathrm{MnB}_{4}$ \\
\hline Formula weight $(\mathrm{g} / \mathrm{mol})$ & 98.18 \\
\hline Temperature (K) & $296(2)$ \\
\hline Wavelength $(\AA)$ & 0.7107 \\
\hline Crystal system & Monoclinic \\
\hline Space group & $P 2_{1} / c$ \\
\hline$a(\AA)$ & $5.4759(4)$ \\
\hline$b(\AA)$ & $5.3665(4)$ \\
\hline$c(\AA)$ & $5.5021(4)$ \\
\hline$\beta\left(^{\circ}\right)$ & $115.044(9)$ \\
\hline$V\left(\AA^{3}\right)$ & $146.486(19)$ \\
\hline$Z$ & 4 \\
\hline Calculated density $\left(\mathrm{g} / \mathrm{cm}^{3}\right)$ & 4.452 \\
\hline Linear absorption coefficient $\left(\mathrm{mm}^{-1}\right)$ & 8.319 \\
\hline$F(000)$ & 180 \\
\hline Crystal size $\left(\mathrm{mm}^{3}\right)$ & $0.05 \times 0.04 \times 0.01$ \\
\hline Theta range for data collection (deg) & $4.11-34.57$ \\
\hline Completeness to theta $=27.59^{\circ}$ & $100.0 \%$ \\
\hline \multirow[t]{3}{*}{ Index ranges } & $-8<h<8$ \\
\hline & $-8<k<7$ \\
\hline & $-8<l<8$ \\
\hline Reflections collected & 2122 \\
\hline Independent reflections $/ R_{\text {int }}$ & $593 / 0.0467$ \\
\hline Max. and min. transmission & 1.00000 and 0.78298 \\
\hline Refinement method & Full matrix least squares on $F^{2}$ \\
\hline Data/restraints/parameters & $593 / 0 / 34$ \\
\hline Goodness of fit on $F^{2}$ & 1.043 \\
\hline Final $R$ indices $[I>2 \sigma(I)]$ & $R_{1}=0.0376, w R_{2}=0.0731$ \\
\hline$R$ indices (all data) & $R_{1}=0.0652, w R_{2}=0.0813$ \\
\hline Largest diff. peak and hole $\left(e / \AA^{3}\right)$ & 0.728 and -0.911 \\
\hline
\end{tabular}

was determined by the shift of the ruby luminescence line. The data were collected using a PerkinElmer XRD1621 detector and two-dimensional (2D) X-ray images were integrated using the FIT2D program [26].

\section{E. Transmission electron microscopy}

The sample for transmission electron microscopy (TEM) was prepared by crushing the material in an agate mortar under ethanol and depositing drops of the suspension on a holey carbon grid. The electron diffraction (ED) patterns and highresolution TEM (HRTEM) images have been acquired using a FEI Tecnai G2 microscope operated at $200 \mathrm{kV}$. Theoretical HRTEM images were calculated using the JEMS software.

\section{F. Hardness measurements}

Vickers hardness $\left(H_{v}\right)$ was measured using a microhardness tester (M-400-G2, LECO Corporation) under loads of $0.5 \mathrm{kgf}$ $(4.9 \mathrm{~N}), 1 \mathrm{kgf}(9.8 \mathrm{~N})$, and $1.5 \mathrm{kgf}(14.7 \mathrm{~N})$.

Nanoindentation (NI) measurements were performed using the electrostatic transducer of the UBI 1 Hysitron triboscope with a pristine diamond $90^{\circ}$ cube corner tip. We made several single (trapezoid) and multi-indentation measurements at three different areas on the sample with target loads 1.5, 2.5, 3.5, 4.5 , and $6 \mathrm{mN}$. 

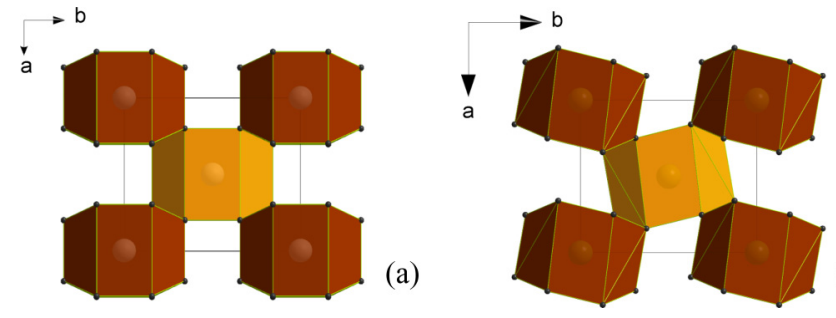

(b)

FIG. 1. (Color online) A comparison of the crystal structure of $\mathrm{MnB}_{4}$ proposed by Andersson [16] (a), and that of $\mathrm{FeB}_{4}$ (Ref. [6]) structure (b). In both cases $M \mathrm{~B} 12$ polyhedra pack in columns, each one is shifted on a $c / 2$ distance along the $c$ direction with respect to its four nearest neighbors (light and dark polyhedra); however, a distortion of the three-dimensional boron network is different.

\section{G. Magnetic and thermodynamic measurements}

The magnetic susceptibility was measured on small polycrystalline pieces of $\mathrm{MnB}_{4}$ using the Quantum Design MPMS superconducting quantum interference device (SQUID) magnetometer. The data were collected at temperatures of $2-380 \mathrm{~K}$ in magnetic fields up to $5 \mathrm{~T}$. The heat capacity was measured by a relaxation technique with the Quantum Design PPMS in the temperature range $1.8-200 \mathrm{~K}$ in fields of 0 and $5 \mathrm{~T}$.

\section{H. Electronic structure calculations}

For electronic structure calculations, we used the fullpotential local-orbital FPLO code [27] and the standard Perdew-Wang local density approximation (LDA) [28] for the exchange-correlation potential. The symmetry-irreducible part of the first Brillouin zone was sampled by a dense $k$ mesh of 518 points. The convergence with respect to the $k$ mesh was carefully checked.

\section{RESULTS}

\section{A. Crystal structure}

Based on powder $\mathrm{x}$-ray diffraction data the crystal structure of $\mathrm{MnB}_{4}$ was initially established as monoclinic [16,17] (space group $C 2 / \mathrm{m})$. The reported unit cell parameters were $a=$ 5.5029(3), $b=5.3669(3), c=2.9487(2) \AA, \beta=122.710(5)^{\circ}$, and the structure was described as a three-dimensional boron network with Mn atoms inside the voids [17]. Each Mn atom is surrounded by 12 boron atoms and the distorted MnB12 polyhedra pack in columns parallel to the $c$ direction [Fig. 1(a)] so that the metal atoms form one-dimensional chains with uniform Mn-Mn distances of 2.9487(2) A. Every column of MnB12 polyhedra is shifted with respect to the four nearest ones over half of the $c$ parameter. Andersson and Carlsson [17] described the structure of $\mathrm{MnB}_{4}$ as highly similar to that of the orthorhombic $\mathrm{CrB}_{4}$ (space group Immm) [29] with insignificant differences in the atomic arrangement.

Recent ab initio calculations [30] showed that the $M \mathrm{~B}_{4}$ ( $M=\mathrm{Cr}, \mathrm{Mn}, \mathrm{Fe}, \mathrm{Tc}, \mathrm{Ru})$ compounds are more stable if the structures have the symmetry described by the Pnnm space group. Indeed, investigation of the synthesized $\mathrm{CrB}_{4}$ powder [30] by means of the electron and $\mathrm{x}$-ray diffraction confirmed the existence of the orthorhombic (Pnnm) $\mathrm{CrB}_{4}$ phase, whose structure was refined by Knappschneider et al. [14] based on
TABLE II. Atomic coordinates, positions and equivalent isotropic displacement parameters for $\mathrm{MnB}_{4}$.

\begin{tabular}{lccccc}
\hline \hline Atom & Wykoff site & $x$ & $y$ & $z$ & $U_{\text {eq }}{ }^{\mathrm{a}}\left(\AA^{2}\right)$ \\
\hline $\mathrm{Mn}(1)$ & $4 e$ & $0.26817(9)$ & $0.0011(2)$ & $0.27375(8)$ & $0.00465(15)$ \\
$\mathrm{B}(1)$ & $4 e$ & $0.3648(9)$ & $0.1859(8)$ & $0.6378(8)$ & $0.0072(4)^{\mathrm{b}}$ \\
$\mathrm{B}(2)$ & $4 e$ & $0.6699(8)$ & $0.1302(8)$ & $0.3238(8)$ & $0.0067(4)^{\mathrm{b}}$ \\
$\mathrm{B}(3)$ & $4 e$ & $0.8692(9)$ & $0.1822(8)$ & $0.1269(8)$ & $0.0072(4)^{\mathrm{b}}$ \\
$\mathrm{B}(4)$ & $4 e$ & $0.1639(8)$ & $0.1301(8)$ & $0.8405(8)$ & $0.0067(4)^{\mathrm{b}}$ \\
\hline \hline
\end{tabular}

${ }^{\mathrm{a}} U_{\text {eq }}$ is defined as one third of the trace of the orthogonalized $U^{i j}$ tensor.

${ }^{\mathrm{b}}$ Atomic displacement parameters for $\mathrm{B}(1)$ and $\mathrm{B}(3)$ and for $\mathrm{B}(2)$ and $\mathrm{B}(4)$ have been fixed to be equal to each other.

single-crystal x-ray diffraction data. Our recent studies [6] showed that $\mathrm{FeB}_{4}$ has the same crystal structure as $\mathrm{CrB}_{4}$. The unit cell contains three independent atoms: one $M(1)$ atom in the $(0,0,0)$ position at the center of inversion and two boron atoms, $\mathrm{B}(1)$ and $\mathrm{B}(2)$ in the $4 g$ position. The major difference from the Immm structure (used by Andersson and Lundstroem [29] to describe the $\mathrm{CrB}_{4}$ structure) is a distortion of the threedimensional boron network [Fig. 1(b)]. Metal-metal distances in the Pnnm structures of $\mathrm{CrB}_{4}$ and $\mathrm{FeB}_{4}$ are 2.8659(1) and 2.9991(2) A, respectively.

For $\mathrm{MnB}_{4}$ we could expect the orthorhombic Pnnm crystal structure, but according to our findings, the $\beta$ angle slightly differs from $90^{\circ}$. The distortion reduces the symmetry of the unit cell to monoclinic $\left(P 2_{1} / n\right)$ with $a=4.6306(3), b=$ 5.3657(4), $c=2.9482(2) \AA$, and $\beta=90.307(6)^{\circ}$. Moreover, in addition to the main reflections of the Pnnm subcell we have observed weak superstructure reflections corresponding to the $\mathbf{k}=(1 / 2,0,1 / 2)$ propagation vector. Using the following transformation, $\mathbf{a}^{\prime}=\mathbf{a}+\mathbf{c}, \mathbf{b}^{\prime}=-\mathbf{b}, \mathbf{c}^{\prime}=\mathbf{a}-\mathbf{c}$, it was possible to index all reflections in the monoclinic unit cell $\left(P 2_{1} / c\right)$ with $a=5.4759(4), b=5.3665(4), c=5.5021(4) \AA$, and $\beta=$ $115.044(9)^{\circ}$. The unit cell of the $\mathrm{MnB}_{4}$ structure contains five independent atoms $[\mathrm{Mn}(1)$ and $\mathrm{B}(1-4)$ atoms, see Table II].

The obtained structure (Fig. 2) can be described in terms of the parent Pnnm cell plus a symmetry breaking structural distortion. The analysis of symmetry modes performed with the program AMPLIMODES [31,32] has shown that the $P 2_{1} / c$ distortion decomposes into two distortion modes of different symmetry corresponding to the irreducible representations (irreps) GM4+ and U1-.

The U1- irrep, associated with the k-vector $(1 / 2,0,1 / 2)$ occurs as a primary mode for this distortion. It involves the displacements of $\mathrm{Mn}$ atoms along [101], thus resulting in two different Mn-Mn distances, namely 2.7004(6) and 3.1953(7) $\AA$ [Fig. 2(a)]. This effect can be understood as a Peierls distortion [33] of the Mn chains. In Fig. 3, we compare local density approximation (LDA) densities of states (DOS) calculated for the monoclinic $P 2_{1} / c$ structure and for the orthorhombic Pnnm substructure, which is constructed as an "average" of the experimental $\mathrm{CrB}_{4}$ and $\mathrm{FeB}_{4}$ structures (averaged lattice parameters and atomic positions). In the orthorhombic structure, the Fermi level of $\mathrm{MnB}_{4}$ would match the peak in the DOS, thus destabilizing the system. This effect is mitigated by a conventional Peierls distortion that splits the 

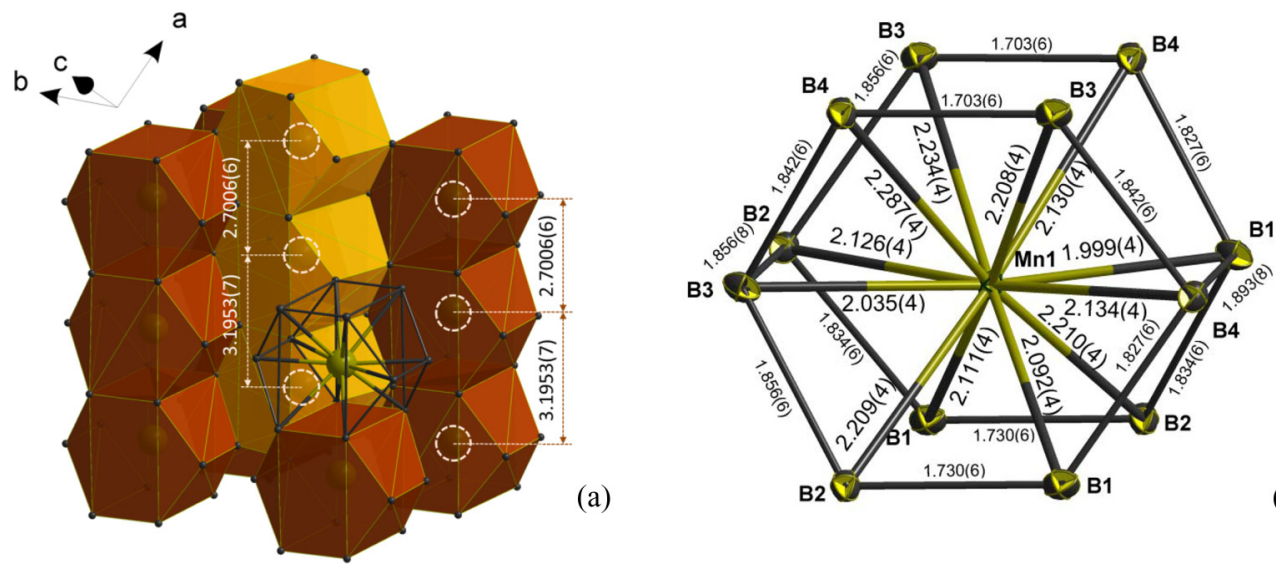

(b)

FIG. 2. (Color online) Structure of $\mathrm{MnB}_{4}$. MnB12 polyhedra pack in columns along [101] direction with alternating Mn-Mn distances of

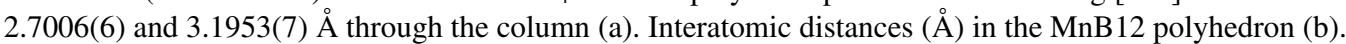

Mn chains with uniform Mn-Mn distances of about $2.93 \AA$ into dimerized Mn chains with alternating $\mathrm{Mn}-\mathrm{Mn}$ distances of 2.7004(6) and 3.1953(7) $\AA$ (as revealed by single-crystal $\mathrm{x}$-ray diffraction). This way, the Fermi level falls into a dip of the DOS, which is unusual for transition-metal tetraborides. Indeed, both $\mathrm{CrB}_{4}$ and $\mathrm{FeB}_{4}$ remain orthorhombic and feature a relatively high DOS at the Fermi level, but remain stable with respect to the Peierls distortion.

While the present paper was under review, the same structure was proposed as the ground state structure for $\mathrm{MnB}_{4}$ based on theoretical calculations [34] and determined experimentally [35] for normal-pressure synthesized $\mathrm{MnB}_{4}$ crystals.

The results of our TEM analysis are in agreement with the single-crystal XRD. Figure 4 shows the ED patterns of $\mathrm{MnB}_{4}$. The patterns were indexed on a primitive monoclinic lattice

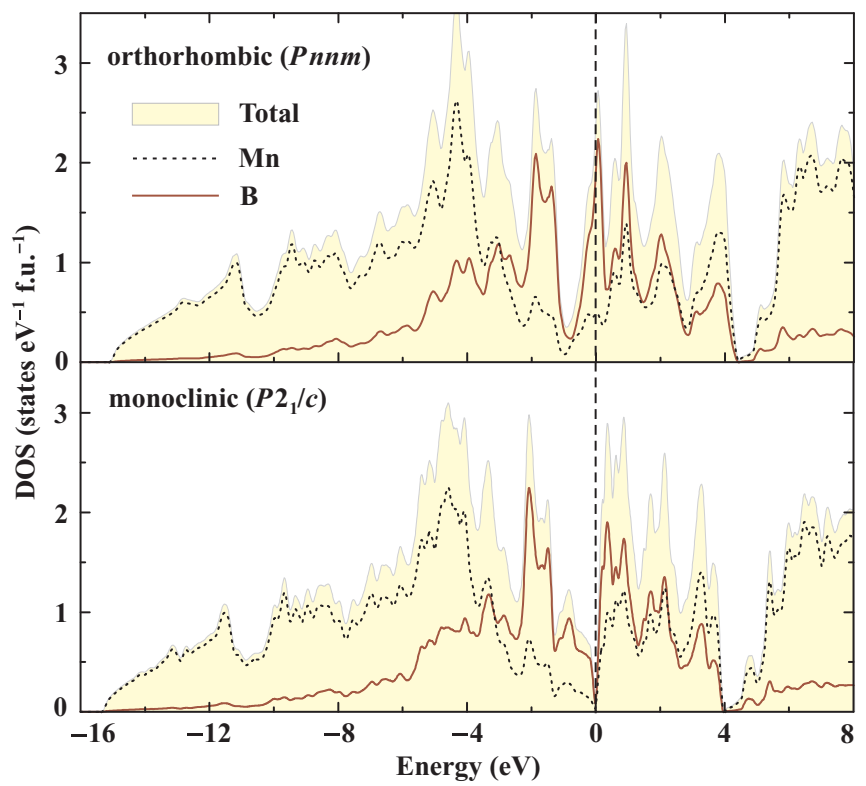

FIG. 3. (Color online) LDA DOS for $\mathrm{MnB}_{4}$ in its fictitious $\mathrm{FeB}_{4}$ like (orthorhombic, top panel) and real (monoclinic, bottom panel) structures. The monoclinic distortion shifts the Fermi level away from the DOS maximum and nearly opens a gap. with the cell parameters $a \approx 5.5 \AA, b \approx 5.4 \AA, c \approx 5.5 \AA$, $\beta \approx 115^{\circ}$, in agreement with the crystal structure determined from x-ray diffraction data. The [010] ED pattern [Fig. 4(d)] demonstrates apparent orthorhombic symmetry which results from a superposition of two mirror twinned variants of the monoclinic structure, shown in Figs. 4(e) and 4(f). Taking into account twinning, the reflection conditions can be determined as $h 0 l: l=2 n$ and $0 k 0: k=2 n$ [Figs. 4(a)-4(d)] that confirm the space group $P 2_{1} / c$. The forbidden $0 k 0, k$-odd reflections on the [001] and [100] ED patterns are caused by multiple diffraction as confirmed by the absence of these forbidden reflections in the [-101] ED pattern.

The [010] HRTEM image in Fig. 5 demonstrates that the $\mathrm{MnB}_{4}$ crystal is almost free of extended defects. At these particular imaging conditions, the bright dots in the image correspond to projections of the Mn columns. The simulated HRTEM image, calculated with the crystal structure refined from single-crystal $\mathrm{x}$-ray diffraction data, is in excellent agreement with the experimental one. Figure 6 demonstrates a [010] HRTEM image of two twinned domains of the monoclinic $\mathrm{MnB}_{4}$ structure. In spite of the coherent twin, no well-defined twin boundary separating the two domains is detected along this projection.
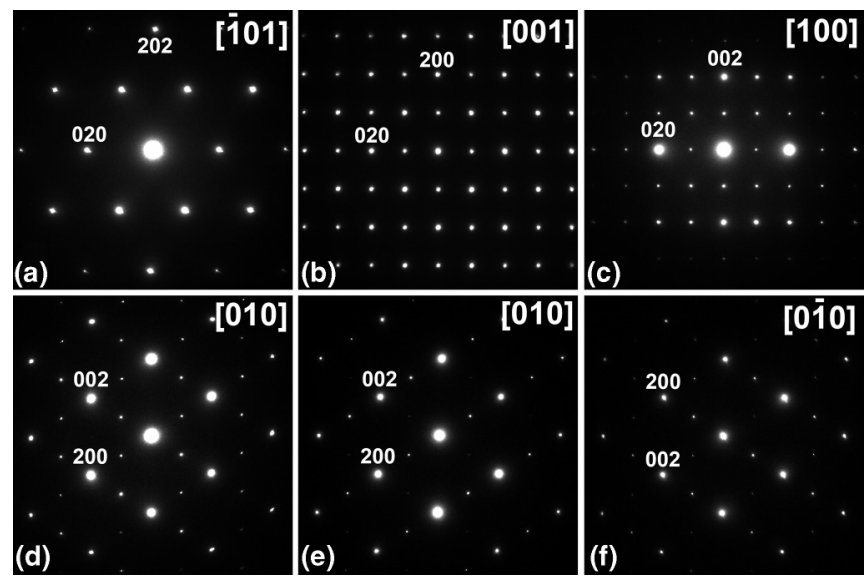

FIG. 4. Electron diffraction patterns of $\mathrm{MnB}_{4}$. The [010] ED pattern (d) is a superposition of two twinned variants (e) and (f). 


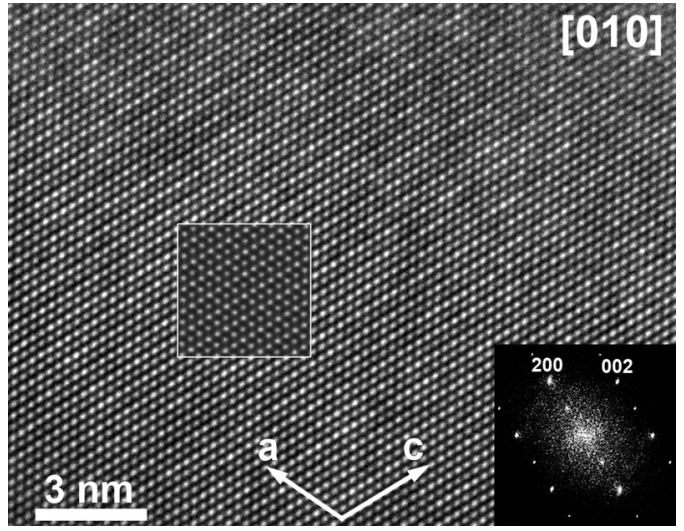

FIG. 5. [010] HRTEM image of a single domain of $\mathrm{MnB}_{4}$ and its Fourier transform. The insert shows a calculated HRTEM image (defocus $f=7 \mathrm{~nm}$, thickness $t=4.8 \mathrm{~nm}$ ).

\section{B. Mechanical properties}

Figure 2(b) shows interatomic distances in $\mathrm{MnB}_{4}$. The B-B distance of 1.703(6) $\AA$ is the shortest among the $M \mathrm{~B}_{4}(M=$ $\mathrm{Cr}, \mathrm{Fe}, \mathrm{Mn}$ ) compounds with similar crystal structures (see Table III). According to Refs. [14] and [6], short B-B bonds are responsible for high hardness and low compressibility of $\mathrm{CrB}_{4}$ and $\mathrm{FeB}_{4}$; therefore we could expect similar properties in $\mathrm{MnB}_{4}$.

The variations of the volume and lattice parameters of $\mathrm{MnB}_{4}$ with pressure up to $25 \mathrm{GPa}$ are presented in Fig. 7. The fit of the pressure-volume data with the third-order Birch-Murnaghan equation of state gave a bulk modulus of $K=254$ (9) GPa and $K^{\prime}=4.4$ [Fig. 7(a)]. The value of the bulk modulus is very close to that reported for $\mathrm{FeB}_{4}, 252(5) \mathrm{GPa}$ [6]. Considerable anisotropy of the compressibility is also similar to that observed in $\mathrm{FeB}_{4}$ [6]. Along the $b$ direction [Fig. 7(b)] the material is almost as incompressible as diamond [36]; this

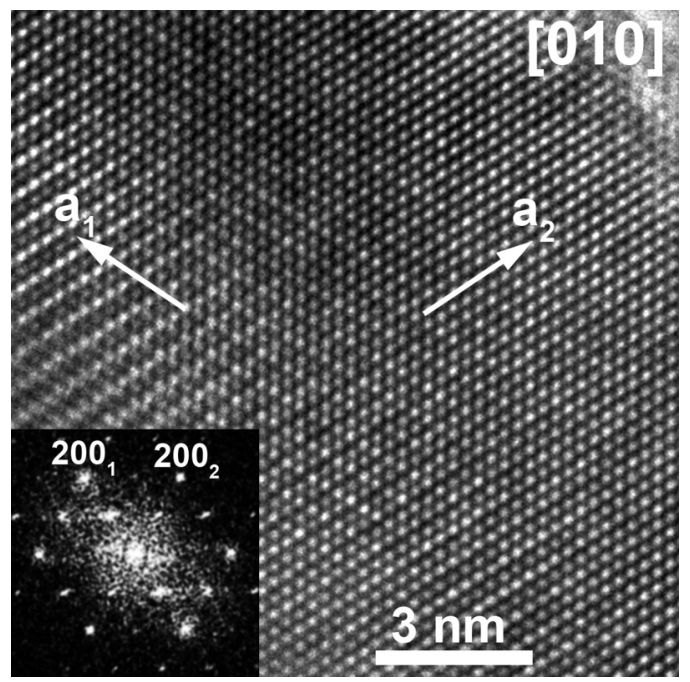

FIG. 6. [010] HRTEM image of two twinned domains of the $\mathrm{MnB}_{4}$ structure (at the left and right side of the image, respectively) and corresponding Fourier transform showing two mirror-related orientations of the $a$ axis of the domains. No well-defined twin boundary is visible along this zone axis.
TABLE III. Bond lengths in $M \mathrm{~B}_{4}(M=\mathrm{Mn}, \mathrm{Cr}, \mathrm{Fe})$ possessing similar structures.

\begin{tabular}{lccc}
\hline \hline Metal boride & $M$-B distances $(\AA)$ & B-B distances $(\AA)$ & Reference \\
\hline $\mathrm{MnB}_{4}$ & $1.999(4)-2.310(4)$ & $1.703(6)-1.893(8)$ & This work \\
$\mathrm{CrB}_{4}$ & $2.053(4)$ & $1.743(6)$ & {$[14]$} \\
& $2.153(4)$ & $1.835(4)$ & \\
& $2.178(3)$ & $1.868(6)$ & \\
& $2.261(3)$ & & \\
& $2.009(4)$ & $1.714(6)$ & \\
$\mathrm{FeB}_{4}$ & $2.109(4)$ & $1.8443(3)$ & \\
& $2.136(3)$ & $1.894(6)$ & \\
& $2.266(3)$ & & \\
\hline \hline
\end{tabular}

can be linked to the very short B-B bond [Fig. 2(b), Table III] along the $b$ axis.

The Vickers hardness of the monoclinic $\mathrm{MnB}_{4}$ was found to be $37.4 \mathrm{GPa}$ at a load of $9.8 \mathrm{~N}$ and $34.6 \mathrm{GPa}$ at $14.7 \mathrm{~N}$; this is larger than that of the $5 d$ transition-metal borides, $\mathrm{WB}_{4}$ (28.1 GPa [12] or 31.8 $\mathrm{GPa}[15]$ at $4.9 \mathrm{~N}), \mathrm{ReB}_{2}(18 \mathrm{GPa}$ [13] at 9.8 N, 26.0-32.5 GPa [9] or 26.6 GPa [12] at 4.9 N), $\mathrm{OsB}_{2}$ (19.6 GPa [8] or 16.8 GPa [12] at $4.9 \mathrm{~N})$. Nanoindentation measurements resulted in the average hardness of $30.7 \pm$ $2.3 \mathrm{GPa}$ and the average indentation modulus of $415 \pm 30 \mathrm{GPa}$. Thus $\mathrm{MnB}_{4}$ is a fairly hard, but not superhard material. It is brittle, as indicated by the typical pop-ins and also cracks appearing sometimes after indentation and visible in the atomic force microscopy (AFM) images.

\section{Magnetic properties and electronic structure}

Magnetic susceptibility of $\mathrm{MnB}_{4}$ reveals a weak ferromagnetic signal at low temperatures (Fig. 8). Above 150$200 \mathrm{~K}, \mathrm{MnB}_{4}$ shows the paramagnetic Curie-Weiss behavior with the effective magnetic moment of 1.6-1.7 $\mu_{B}$ and the ferromagnetic Weiss temperature of $\theta \sim 90 \mathrm{~K}$ according to

$$
\chi=C /(T-\theta) .
$$

In Fig. 8 we show magnetic susceptibility data collected on two different samples which are both single phase according to XRD and WDX. While the high-temperature regions match quite well, the behavior at low temperatures is remarkably different and shows a variable magnitude of the ferromagnetic signal. Magnetization isotherms measured at $2 \mathrm{~K}$ further show a small, but variable net moment (Fig. 9). Therefore, we conclude that $\mathrm{MnB}_{4}$ reveals ferromagnetic spin correlations evidenced by the positive $\theta$ value extracted from the robust high-temperature data. On the other hand, the low-temperature ferromagnetism of our samples (the net moment observed at low temperatures) appears to be extrinsic. Note also that no abrupt phase transition, such as ferromagnetic ordering, can be seen in the magnetization data.

Considering the LDA electronic structure of the stoichiometric monoclinic $\mathrm{MnB}_{4}$ (Fig. 3, bottom), one would expect a weak paramagnetic or even a diamagnetic behavior of this compound, because the Fermi level falls into a dip in the DOS formed upon the Peierls distortion, hence the number of states at the Fermi level is extremely low, only $N\left(E_{F}\right)$ 

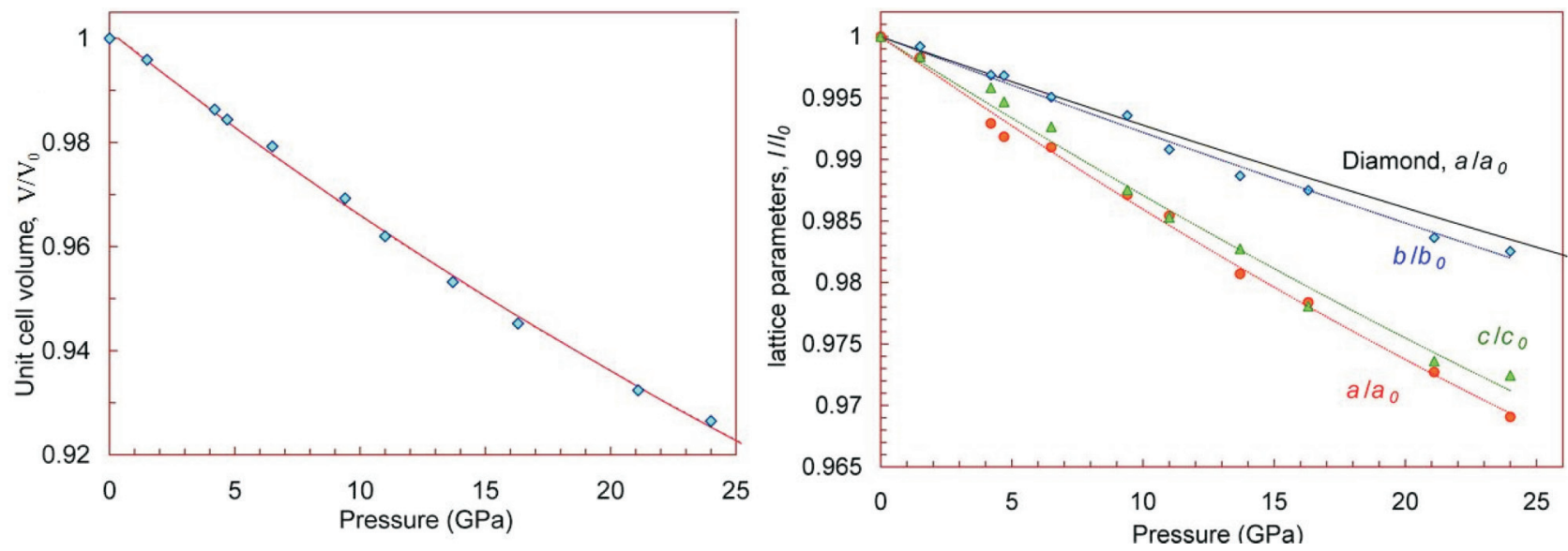

FIG. 7. (Color online) Compressibility of $\mathrm{MnB}_{4}$. (a) The pressure dependence of the relative unit cell volume based on powder synchrotron $\mathrm{x}$-ray diffraction data. Solid line corresponds to the fit of the pressure-volume data with the third-order Birch-Murnaghan equation of state, which gave the bulk modulus $K=254(9) \mathrm{GPa}$ and $K^{\prime}=4.4$. (b) The relative changes of the unit cell parameters as a function of pressure. The stiffness of the $\mathrm{MnB}_{4}$ structure along the $b$ direction is almost the same as that of diamond (continuous black line according to Ref. [36]).

$\sim 0.08 \mathrm{eV}^{-1} /$ f.u., compared to $N\left(E_{F}\right) \sim 1.0 \mathrm{eV}^{-1} /$ f.u. in $\mathrm{FeB}_{4}$. Surprisingly, our low-temperature heat-capacity measurements revealed a large electronic contribution to the specific heat. In the $15-30 \mathrm{~K}$ temperature range, the heat capacity can be fitted to the conventional expression for metals:

$$
C_{P}(T)=\gamma T+\beta T^{3},
$$

where the first and second terms stand for the electronic and lattice contributions, respectively (Fig. 10). The fit yields $\gamma=$ $10.1 \mathrm{~mJ} \mathrm{~mol}^{-1} \mathrm{~K}^{-2}$ and $\beta=0.012 \mathrm{~mJ} \mathrm{~mol}^{-1} \mathrm{~K}^{-4}$. Below $15 \mathrm{~K}$, an additional contribution to the specific heat is clearly seen in Fig. 10. This contribution does not change in the applied field and may reflect nonmagnetic impurity states leading to a series of Schottky anomalies. Its exact nature requires further investigation.

The $\beta$ and $\gamma$ parameters for $\mathrm{MnB}_{4}$ are akin to those for $\mathrm{FeB}_{4}$, where we previously reported $\gamma=10.2 \mathrm{~mJ} \mathrm{~mol}^{-1} \mathrm{~K}^{-2}$ and $\beta=0.025 \mathrm{~mJ} \mathrm{~mol}^{-1} \mathrm{~K}^{-4}$ (Ref. [6]). Compared to

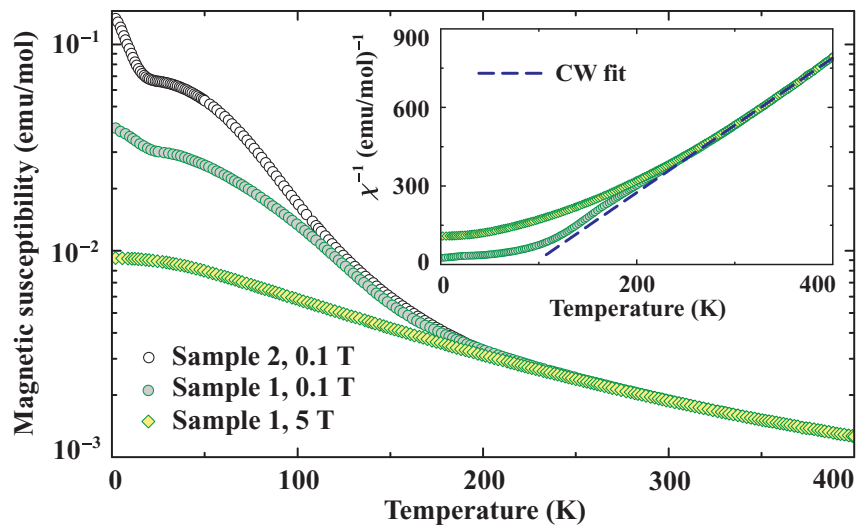

FIG. 8. (Color online) Magnetic susceptibility of $\mathrm{MnB}_{4}$ measured on two different samples. At high temperatures, the susceptibility is nearly sample independent and yields the Curie-Weiss (CW) parameters of $\mu_{\text {eff }} \sim 1.7 \mu_{\mathrm{B}}$ and $\theta \sim 90 \mathrm{~K}$ (see inset). At low temperatures, the susceptibility is strongly sample dependent indicating a variable net moment, which is most likely extrinsic. superhard $\mathrm{FeB}_{4}$, the $\beta$ value in $\mathrm{MnB}_{4}$ is reduced by a factor of 2 , which is well in line with our finding that $\mathrm{MnB}_{4}$ is hard but not superhard. Its effective Debye temperature is $\theta_{D} \sim 540 \mathrm{~K}$, and the $\beta T^{3}$ behavior of the lattice specific heat persists up to at least $30 \mathrm{~K}$.

Regarding the electronic contribution to the specific heat, the $\gamma$ values of about $10 \mathrm{~mJ} \mathrm{~mol}^{-1} \mathrm{~K}^{-2}$ for $\mathrm{MnB}_{4}$ and $\mathrm{FeB}_{4}$ are remarkably similar. For a simple metal, they would imply a high density of states at the Fermi level, $N\left(E_{F}\right) \sim 4.3$ states $\mathrm{eV}^{-1}$ f.u. ${ }^{-1}$, which is four times higher than the LDA estimate for $\mathrm{FeB}_{4}\left(\sim 1.0\right.$ states $\mathrm{eV}^{-1}$ f.u. ${ }^{-1}$, Ref. [5]) and 50 times higher than the LDA estimate for $\mathrm{MnB}_{4}\left(\sim 0.08\right.$ states $\mathrm{eV}^{-1}$ f.u. $\left.{ }^{-1}\right)$. Apparently, there is a strong renormalization of $\gamma$ in transition-metal tetraborides, yet in $\mathrm{MnB}_{4}$ this effect is particularly strong. Possible reasons behind it will be discussed below.

The high value of $\gamma$ suggests that at least at low temperatures $\mathrm{MnB}_{4}$ features a large number of charge carriers and should be metallic. While the small size of the available samples prevents us from performing the resistivity measurements, we note that already the large $\gamma$ value contradicts the

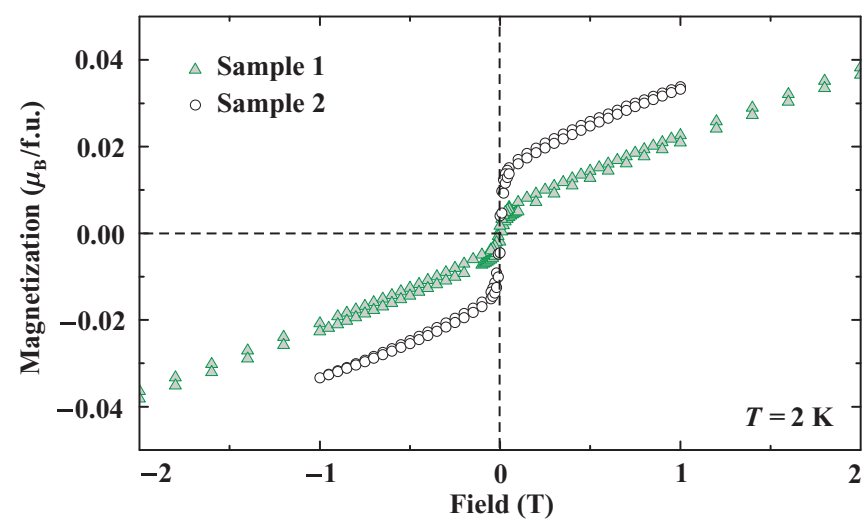

FIG. 9. (Color online) Magnetization curves of two $\mathrm{MnB}_{4}$ samples measured at $2 \mathrm{~K}$. Note the different net moments and the similar slope of the linear part. 


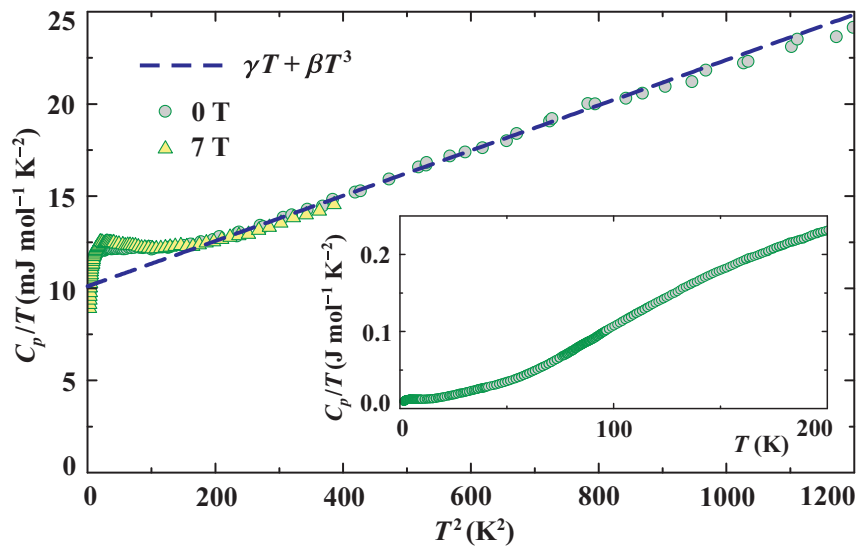

FIG. 10. (Color online) The specific heat of $\mathrm{MnB}_{4}$ measured in the applied fields of $0 \mathrm{~T}$ (circles) and $7 \mathrm{~T}$ (triangles). The line shows the fit with Eq. (2). The inset displays the smooth temperature dependence of the specific heat in a broad temperature range up to $200 \mathrm{~K}$.

simple scenario of a Peierls distortion that would drastically reduce the number of states at the Fermi level (Fig. 3, bottom). Moreover, ferromagnetic spin correlations cannot be understood on the basis of LDA results, because the Peierls dimerization typically leads to a nonmagnetic state. Indeed, spin-polarized the local spin-density approximation (LSDA) calculations for $\mathrm{MnB}_{4}$ converge to a nonmagnetic solution, which contradicts the sizable effective moment and ferromagnetic spin correlations (positive $\theta$ value) observed in our magnetization measurements (Fig. 8).

The discrepancies between the nonmagnetic, nearly insulating LDA scenario and the experimental ferromagnetic metallic behavior can be ascribed to several effects. First, tiny deviations from the ideal $\mathrm{MnB}_{4}$ stoichiometry may push the Fermi level out of the dip and increase the number of states at the Fermi level. However, this effect is by far insufficient to reproduce our results. A tentative modeling of the nonstoichiometric $\mathrm{MnB}_{4}$ within the virtual crystal approximation (VCA), that basically changes the charge on the Mn site and shifts the Fermi level toward lower or higher energies, fails to account for ferromagnetic spin correlations: The system remains nonmagnetic even at the $10 \%$ doping level, while the composition of our samples is established as stoichiometric $\mathrm{MnB}_{4}$ with less than $1 \%$ uncertainty. A more plausible explanation would be an increased tendency to electron localization on the Mn sites. This tendency can be reproduced by the LSDA $+U$ method that adds a meanfield Hubbard-like energy term and mimics the effect of the on-site Coulomb repulsion $U$. Although originally designed for insulators, the LSDA $+U$ method can be also applied to metallic systems and provides a rough guess on the behavior of correlated metals [37].

Here, we used LSDA $+U$ with the on-site Coulomb repulsion $U=3 \mathrm{eV}$ and Hund's coupling $J=0.5 \mathrm{eV}$, which were taken about twice lower than standard estimates for strongly correlated insulating Mn oxides $(U=5-6 \mathrm{eV}$, $J=1 \mathrm{eV}$, Refs. [38,33]). This way, we are able to stabilize a ferromagnetic solution with a small moment of about $0.6 \mu_{B}$ on Mn atoms (Fig. 11). This moment is still much lower than the high-temperature paramagnetic effective moment of about

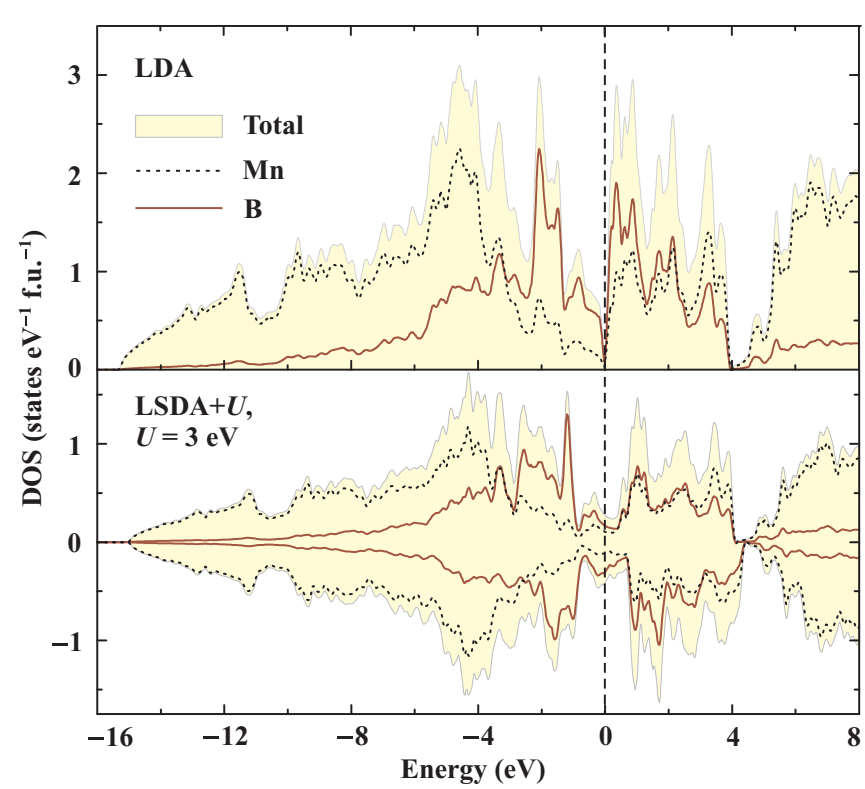

FIG. 11. (Color online) Electronic structure of monoclinic $\mathrm{MnB}_{4}$ calculated within LDA (top panel) and LSDA $+U$ with $U=3 \mathrm{eV}$ (bottom).

$1.7 \mu_{B}$. However, these two moments are not expected to match, because the LSDA $+U$ result pertains to the ordered moment at zero temperature, while the effective moment is the fluctuating moment at high temperatures. In fact, our calculated moment is in the same range as the ordered moment in Mn-based weak ferromagnets, such as MnSi: $\mu=0.4 \mu_{B}$ (Ref. [39]). Moreover, we find a sizable density of states at the Fermi level, $N\left(E_{F}\right) \sim 0.7$ states $\mathrm{eV}^{-1}$ f.u. ${ }^{-1}$ (Fig. 11) that now approaches $N\left(E_{F}\right) \sim 1.0$ states $\mathrm{eV}^{-1}$ f.u. ${ }^{-1}$ for $\mathrm{FeB}_{4}$ and better matches the experimental value of $\gamma$, although a large renormalization is still required.

\section{DISCUSSION}

$\mathrm{MnB}_{4}$ has its distinct position in the family of transitionmetal tetraborides. Both $\mathrm{CrB}_{4}$ and $\mathrm{FeB}_{4}$ are orthorhombic and, in general, well described by standard LDA that accurately predicted the orthorhombic crystal structure of $\mathrm{FeB}_{4}$ and even the superconductivity of this compound [5,6]. In $\mathrm{MnB}_{4}$, the electron count is such that the Fermi level matches the maximum of the density of states. Then the orthorhombic structure becomes unstable and undergoes a monoclinic distortion. We ascribe this effect to a Peierls distortion [33], because in the monoclinic structure the Mn chains are dimerized, and the Fermi level falls into a dip in the density of states, which is strongly reminiscent of a band gap observed in other Peierls-distorted systems [40,41]. Therefore, $\mathrm{MnB}_{4}$ could even be similar to narrow-gap intermetallic compounds, such as $\mathrm{FeGa}_{3}$ (Ref. [42]). An important difference though is that in those compounds the band gap would typically open because of the strong hybridization (formation of separated bonding and antibonding states) between the transition-metal and $p$-element orbitals. In $\mathrm{MnB}_{4}$, the dip in the LDA DOS arises from the $\mathrm{Mn}-\mathrm{Mn}$ interactions, while the mixing with the B states keeps the system metallic and provides a small yet nonzero number of states at the Fermi level. 
Surprisingly, our experimental data are not consistent with this simple dimerization picture, because $\mathrm{MnB}_{4}$ shows a large electronic contribution to the specific heat and a sizable hightemperature paramagnetic moment with clear signatures of ferromagnetic spin correlations. Phenomenologically, $\mathrm{MnB}_{4}$ is similar to Mn-based ferromagnets, such as MnSi (compare, for example, the high-temperature paramagnetic moments of $\sim 1.7$ and $2.3 \mu_{B}$ (Ref. [39]), respectively), with the only exception that $\mathrm{MnB}_{4}$ does not show any clear signature of the long-range ferromagnetic order. We have shown that moderate electronic correlations may reconcile experimental observations with computational results and render $\mathrm{MnB}_{4}$ ferromagnetic. However, the origin of these correlations is presently unclear, and the absence of the long-range magnetic ordering despite sizable ferromagnetic spin correlations remains an open problem as well.

\section{v. CONCLUSION}

The high-pressure high-temperature synthesis technique enabled us to synthesize high-quality single crystals of manganese tetraboride, $\mathrm{MnB}_{4}$. Single-crystal synchrotron x-ray diffraction data allowed the refinement of its crystal structure, which revealed dimerized Mn chains with alternating Mn-Mn distances, which were not identified in previous powder XRD investigations of polycrystalline $\mathrm{MnB}_{4}$ samples. We explained this phenomenon by a Peierls distortion, which reduces the symmetry of $\mathrm{MnB}_{4}$ to monoclinic, compared to the orthorhombic symmetry of otherwise similar $\mathrm{CrB}_{4}$ and $\mathrm{FeB}_{4}$ structures.
Mechanical property measurements revealed the high bulk modulus [254(9) GPa], strong anisotropy in compressibility (with the stiffness comparable to that of diamond, along the $b$ axis), and very high hardness (35-37 GPa) approaching that of superhard materials. Our experimental studies provide previously unavailable data on magnetic properties of $\mathrm{MnB}_{4}$. The latter, complemented with our theoretical consideration on the electronic properties of $\mathrm{MnB}_{4}$, allowed us to conclude that the relatively simple crystal structure with a well-defined and well-understood Peierls distortion hosts remarkably complex and even enigmatic low-temperature physics. Current efforts in the high-pressure synthesis should eventually result in the preparation of larger samples that would facilitate further studies on the electronic structure and magnetism of this interesting material.

\section{ACKNOWLEDGMENTS}

H.G. gratefully acknowledges financial support of the Alexander von Humboldt Foundation. The work was supported by the German Research Foundation (DFG). N.D. thanks DFG for financial support through the Heisenberg Program and the DFG Project No. DU 954-8/1. A.T. was funded by the Mobilitas program of the ESF (Grant No. MTT77). A.T. acknowledges fruitful discussions with Christoph Geibel and experimental support by Deepa Kasinathan. G.V.T. acknowledges the European Research Council, ERC Grant No. 246791-COUNTATOMS. Portions of this research were carried out at the light source PETRA III at DESY, a member of the Helmholtz Association (HGF).
[1] K. C. Buschveck, Boron Compounds, Elemental Boron and Boron Carbides 13, Gmelin Handbook of Inorganic and Organometallic Chemistry (Springer-Verlag, Berlin, 1981).

[2] B. Albert and H. Hillebrecht, Angew. Chem. Int. Ed. 48, 8640 (2009).

[3] M. M. Balakrishnarajan and R. Hoffmann, J. Am. Chem. Soc. 126, 13119 (2004).

[4] A. Hermann, A. McSorley, N. W. Ashcroft, and R. Hoffmann, J. Am. Chem. Soc. 134, 18606 (2012).

[5] A. N. Kolmogorov, S. Shah, E. R. Margine, A. F. Bialon, T. Hammerschmidt, and R. Drautz, Phys. Rev. Lett. 105, 217003 (2010)

[6] H. Gou, N. Dubrovinskaia, E. Bykova, A. A. Tsirlin, D. Kasinathan, W. Schnelle, A. Richter, M. Merlini, M. Hanfland, A. M. Abakumov, D. Batuk, G. Van Tendeloo, Y. Nakajima, A. N. Kolmogorov, and L. Dubrovinsky, Phys. Rev. Lett. 111, 157002 (2013).

[7] J. B. Levine, S. H. Tolbert, and R. B. Kaner, Adv. Funct. Mater. 19, 3519 (2009).

[8] R. W. Cumberland, M. B. Weinberger, J. J. Gilman, S. M. Clark, S. H. Tolbert, and R. B. Kaner, J. Am. Chem. Soc. 127, 7264 (2005).

[9] H.-Y. Chung, M. B. Weinberger, J. B. Levine, A. Kavner, J.-M. Yang, S. H. Tolbert, and R. B. Kaner, Science 316, 436 (2007).

[10] R. B. Kaner, J. J. Gilman, and S. H. Tolbert, Science 308, 1268 (2005).
[11] N. Dubrovinskaia, L. Dubrovinsky, and V. L. Solozhenko, Science 318, 1550 (2007).

[12] Q. F. Gu, G. Krauss, and W. Steurer, Adv. Mater. 20, 3620 (2008).

[13] J. Q. Qin, D. W. He, J. H. Wang, L. M. Fang, L. Lei, Y. J. Li, J. Hu, Z. L. Kou, and Y. Bi, Adv. Mater. 20, 4780 (2008).

[14] A. Knappschneider, C. Litterscheid, D. Dzivenko, J. A. Kurzman, R. Seshadri, N. Wagner, J. Beck, R. Riedel, and B. Albert, Inorg. Chem. 52, 540 (2013).

[15] R. Mohammadi, A. T. Lech, M. Xie, B. E. Yeung, S. H. Tolbert, and R. B. Kaner, Proc. Natl. Acad. Sci. USA 108, 10958 (2011).

[16] S. Andersson, Acta Chem. Scand. 23, 687 (1969).

[17] S. Andersson and J.-O. Carlsson, Acta Chem. Scand. 24, 1791 (1970).

[18] E. Ito, in Treatise on Geophysics, edited by G. D. Price (Elsevier, Amsterdam, 2007), Vol. 2, pp. 198-230.

[19] Agilent Technologies, Agilent Technologies UK Ltd., Oxford, UK, Xcalibur CCD system, CRYSALISPRO software system, version 1.171.35.19 (2012).

[20] G. M. Sheldrick, Acta Crystallogr., Sect. A: Found. Crystallogr. 64, 112 (2008).

[21] K. Brandenburg, DIAMOND, Crystal Impact GbR, Bonn, Germany (1999).

[22] A. Belsky, M. Hellenbrandt, V. L. Karen, and P. Luksch, Acta Crystallogr., Sect. B: Struct. Sci. 58, 364 (2002). 
[23] Fachinformationszentrum Karlsruhe, 76344 EggensteinLeopoldshafen, Germany. http://www.fiz-karlsruhe.de/ request_for_deposited_data.html. FAX: $(+49) 7247-808-666$. Email address: crysdata@fiz-karlsruhe.de

[24] A. Kurnosov, I. Kantor, T. Boffa-Ballaran, S. Lindhardt, L. Dubrovinsky, A. Kuznetsov, and B. H. Zehnder, Rev. Sci. Instrum. 79, 045110 (2008).

[25] H. P. Liermann, W. Morgenroth, A. Ehnes, A. Berghauser, B. Winkler, H. Franz, and E. Weckert, J. Phys. Conf. Ser. 215, 012029 (2010).

[26] A. P. Hammersley, S. O. Svensson, M. Hanfland, A. N. Fitch, and D. Hausermann, High Pressure Res. 14, 235 (1996).

[27] K. Koepernik and H. Eschrig, Phys. Rev. B 59, 1743 (1999).

[28] J. P. Perdew and Y. Wang, Phys. Rev. B 45, 13244 (1992).

[29] S. Andersson and T. Lundstroem, Acta Chem. Scand. 22, 3103 (1968).

[30] H. Niu, J. Wang, X. Q. Chen, D. Li, Y. Li, P. Lazar, R. Podloucky, and A. N. Kolmogorov, Phys. Rev. B 85, 144116 (2012).

[31] D. Orobengoa, C. Capillas, M. I. Aroyo, and J. M. Perez-Mato, J. Appl. Cryst. 42, 820 (2009).

[32] J. M. Perez-Mato, D. Orobengoa, and M. I. Aroyo, Acta Crystallogr., Sect. A: Found. Crystallogr. 66, 558 (2010).
[33] Y. Kumagai, A. A. Belik, M. Lilienblum, N. Leo, M. Fiebig, and N. A. Spaldin, Phys. Rev. B 85, 174422 (2012).

[34] A. G. Van Der Geest and A. N. Kolmogorov, arXiv:1310.4157 [cond-mat.mtrl-sci].

[35] A. Knappschneider, C. Litterscheid, N. C. George, J. Brgoch, N. Wagner, J. Beck, J. A. Kurzman, R. Seshadri, and B. Albert, Angew. Chem. Int. Ed. 53, 1684 (2014).

[36] F. Occelli, P. Loubeyre, and R. Letoullec, Nat. Mater. 2, 151 (2003).

[37] A. G. Petukhov, I. I. Mazin, L. Chioncel, and A. I. Lichtenstein, Phys. Rev. B 67, 153106 (2003).

[38] W. G. Yin, D. Volja, and W. Ku, Phys. Rev. Lett. 96, 116405 (2006).

[39] S. M. Stishov, A. E. Petrova, S. Khasanov, G. Kh. Panova, A. A. Shikov, J. C. Lashley, D. Wu, and T. A. Lograsso, Phys. Rev. B 76, 052405 (2007).

[40] A. J. Berlinsky, Rep. Prog. Phys. 42, 1243 (1979).

[41] T. Toriyama, A. Nakao, Y. Yamaki, H. Nakao, Y. Murakami, K. Hasegawa, M. Isobe, Y. Ueda, A. V. Ushakov, D. I. Khomskii, S. V. Streltsov, T. Konishi, and Y. Ohta, Phys. Rev. Lett. 107, 266402 (2011).

[42] U. Häussermann, M. Boström, P. Viklund, Ö. Rapp, and T. Björnängen, J. Solid State Chem. 165, 94 (2002). 